\title{
Performance Study of a Class of Irregular LDPC Codes Based on Their Weight Distribution Analysis
}

\author{
F. Vatta, F. Babich, F. Ellero, M. Noschese, G. Buttazzoni, and M. Comisso \\ Department of Engineering and Architecture, University of Trieste \\ Trieste, Italy \\ e-mail:vatta@units.it, babich@units.it, flavio.ellero@studenti.units.it \\ matteo.noschese@phd.units.it, gbuttazzoni@units.it, mcomisso@units.it
}

\begin{abstract}
This paper investigates the performance of irregular low-density parity-check (LDPC) codes on memoryless BI-AWGN (Binary Input - Additive White Gaussian Noise) channels, with sum-product decoding. Objective of this work is to study the relationship between an LDPC code performance and some parameters specifying the code itself, such as the coefficients of its degree distributions. In fact, these coefficients where shown in Di et al.'s 2006 paper to determine the growth rate of the minimum distance of an LDPC code, which can be only sublinear in the block length in some well defined conditions of the degree distribution pair.
\end{abstract}

\section{INTRODUCTION}

Low Density Parity Check (LDPC) codes are a class of channel block codes, first introduced in 1960 by Robert Gallager in his doctoral dissertation [1], representing the leading edge in modern channel coding. Due to the technical limitations of that age and the quadratic complexity in the block length making the efficient encoder design not trivial, LDPC codes were scarcely considered for almost 30 years, apart from Tanner's generalized LDPC definition and graphical representation, presented in his 1981 paper [2], which was later called Tanner graph. Apparently independent of Gallager's work, LDPC codes were re-invented in the mid 1990s by MacKay [3] and Luby et al. [4]. Since it was shown that LDPC performance can approach the Shannon limit as well as Turbo codes, these codes were quickly included in modern communication standards such as IEEE802.11n [5][7], 802.16e (Wi-MAX), 10G-BaseT Ethernet, and Digital Video Broadcasting. Recently, they were also proposed as component codes of product code structures [8] for the next generation digital terrestrial broadcasting transmission system [9] and were adopted, together with polar codes, by the fifthgeneration $(5 \mathrm{G})$ new radio (NR) standard [10]-[12].

One of the most important classical assumptions in coding theory is the idea of maximizing a code minimum distance to achieve better performances. This idea is based on the fact that the weight distribution of a code determines quite accurately its bit error rate (BER) or frame error rate (FER) performance bounds (at least away from the Shannon capacity) and that

This work is partly supported by the Italian Ministry of University and Research (MIUR) within the project FRA 2018 (University of Trieste, Italy), entitled "Integrazione cUBEsat-Reti 5G: analisi del livello di accesso e sviluppo di sistemi d'antenna". these bounds are dominated by small Hamming codeword weights.

Since LDPC codes are well known "capacity-approaching codes", when iteratively decoded applying low complexity algorithms, it is objective of this work to study the role played by their codewords weight distribution in determining this very good performance, on the basis of the results of Di et al.'s 2006 paper [13]. In particular, the main result of [13] states that, for irregular LDPC codes ensembles, the growth rate of the minimum distance, i.e., whether this growth rate is linear or simply sublinear, depends only on $\lambda^{\prime}(0) \rho^{\prime}(1)$, a quantity which is related to the degree distributions (1) and (2). As stated more precisely in Theorem 1 of [13], if $\lambda^{\prime}(0) \rho^{\prime}(1)>1$, which is the one examined in this paper, the minimum distance grows sublinearly with the block length, otherwise, i.e., if $\lambda^{\prime}(0) \rho^{\prime}(1)<1$, it grows linearly with the block length, with probability at least $1-\ln \frac{1}{\sqrt{1-\lambda^{\prime}(0) \rho^{\prime}(1)}}$, sharpened to $\sqrt{1-\lambda^{\prime}(0) \rho^{\prime}(1)}$ in [14]

Being the parameter $\lambda^{\prime}(0)$ given by the fraction of edges connecting to degree-two v-nodes, i.e., $\lambda_{2}$ (see Section V), this parameter is fundamental in the design of LDPC codes that can reach capacity under iterative decoding, since the achievement of capacity requires $\lambda_{2} \neq 0$ [13]. However, in this latter case, the average number of weight 2 codewords in the ensemble is bounded away from zero, and thus the block error probability might converge to a constant [13].

The paper is organized as follows. In the next section, we recall the fundamental properties of LDPC codes as far as their matrix representation is concerned, whereas in Section III their graphical representation is recalled. In Section IV are recalled the fundamental results of [13] on the weight distribution of LDPC codes and in Section $\mathrm{V}$ are reported the results of the weight distribution analysis. Finally, in Section VI are reported some simulation results and Section VII summarizes the results of the paper.

\section{MATRIX REPRESENTATION OF LDPC CODES}

Although LDPC codes can be generalized to non-binary alphabets, we shall consider, for simplicity, only binary LDPC codes. Since LDPC codes form a class of linear block codes, they may be described as a certain $k$-dimensional subspace 
$\mathcal{C}$ of the vector space $\mathbb{F}_{2}^{n}$ of binary $n$-tuples over the binary field $\mathbb{F}_{2}$. Each codeword $\mathbf{c} \in \mathcal{C}$ may be written as a linear combination of the vectors of a basis $B=\left\{\mathbf{g}_{0}, \mathbf{g}_{1}, \cdots, \mathbf{g}_{k-1}\right\}$ spanning $\mathcal{C}, \mathbf{c}=u_{0} \mathbf{g}_{0}+u_{1} \mathbf{g}_{1}+\ldots+u_{k-1} \mathbf{g}_{k-1}$ for some $\left\{u_{i}\right\}$. In other words, $\mathbf{c}=\mathbf{u} \cdot G$ where $\mathbf{u}=\left[u_{0} u_{1} \cdots u_{k-1}\right]$ and $G$ is the so-called $k \times n$ generator matrix whose rows are the vectors $\left\{\mathbf{g}_{i}\right\}$. The $(n-k)$-dimensional null space $\mathcal{C}^{\perp}$ of $G$ comprises all vectors $\mathbf{x} \in \mathbb{F}_{2}^{n}$ for which $\mathbf{x} \cdot G^{T}=\mathbf{0}$ and is spanned by the basis $B^{\perp}=\left\{\mathbf{h}_{0}, \mathbf{h}_{1}, \cdots, \mathbf{h}_{n-k-1}\right\}$. Thus, for each $\mathbf{c} \in \mathcal{C}, \mathbf{c} \cdot \mathbf{h}_{i}^{T}=0$ for all $i$ or, more compactly, c. $H^{T}=\mathbf{0}$, where $H$ is the so-called $(n-k) \times n$ parity-check matrix, whose rows are the vectors $\left\{\mathbf{h}_{i}\right\}$, and is the generator matrix for the null space $\mathcal{C}^{\perp}$. The parity-check matrix $H$ is so called because it performs $m=n-k$ separate parity checks on a received word.

An LDPC code is a linear block code for which the paritycheck matrix $H$ has a low density of 1's. A regular LDPC code is a linear block code whose parity-check matrix $H$ contains exactly $w_{c} 1$ 's in each column and exactly $w_{r}=w_{c}(n / m)$ 1 's in each row, where $w_{c}<<m$ (equivalently, $w_{r}<<n$ ). The code rate $R=k / n$ is related to these parameters via $R=1-w_{c} / w_{r}$ (this assumes $H$ is full rank). If $H$ is low density, but the number of 1's in each column or row is not constant, then the code is an irregular LDPC code. It is easier to see the sense in which an LDPC code is regular or irregular through its graphical representation.

\section{GRAPHICAL REPRESENTATION OF LDPC CODES}

Tanner considered LDPC codes and showed how they may be represented effectively by a so-called bipartite graph, now called Tanner graph [2]. The Tanner graph of an LDPC code is analogous to the trellis of a convolutional code in that it provides a complete representation of the code and it aids in the description of the decoding algorithm. A bipartite graph is a graph (nodes connected by edges) whose nodes may be separated into two types, and edges may only connect two nodes of different types. The two types of nodes in a Tanner graph are the variable nodes and the check nodes (which we shall call v-nodes and c-nodes, respectively). The Tanner graph of a code is drawn according to the following rule: check node $j$ is connected to variable node $i$ whenever element $h_{i j}$ in $H$ is a 1. One may deduce from this that there are $m=n-k$ check nodes, one for each check equation, and $n$ variable nodes, one for each code bit $c_{i}$. Moreover, the $m$ rows of $H$ specify the $m$ c-node connections and the $n$ columns of $H$ specify the $n$ v-node connections.

For irregular LDPC codes [4], the parameters $w_{c}$ and $w_{r}$ are functions of the column and row numbers and so such notation is not generally adopted in this case. Instead, they are defined by specifying the distribution of the node degrees in their Tanner graphs. In particular, in the edge-perspective degree distribution, $\lambda_{i}$ is the fraction of edges in the Tanner graph connecting to a degree- $i$ variable node, and $\rho_{j}$ is the fraction of edges connecting to a degree- $j$ check node. To specify the degree distribution, the following polynomials are defined:

$$
\begin{aligned}
& \lambda(x)=\sum_{i=2}^{d_{l}} \lambda_{i} x^{i-1} \\
& \rho(x)=\sum_{j=2}^{d_{r}} \rho_{j} x^{j-1}
\end{aligned}
$$

being $d_{l}$ (respectively $d_{r}$ ) the maximum variable (respectively check) node degree.

The $d_{l}$-tuple $\left\{\lambda_{i}\right\}$ and $d_{r}$-tuple $\left\{\rho_{j}\right\}$ both add up to 1 . Given the polynomials $\lambda(x)$ and $\rho(x)$, a $(\lambda, \rho)$-LDPC code of block length $n$ is defined as a linear code with a Tanner graph in $n$ variable nodes such that the edge-perspective variable-node degree distribution is given by $\lambda(x)$ and the edge-perspective check-node degree distribution is given by $\rho(x)$.

The rate of LDPC codes specified by a degree distribution pair $(\lambda, \rho)$ is computed as [18]

$$
r(\lambda, \rho)=1-\frac{\int_{0}^{1} \rho(x) \mathrm{d} x}{\int_{0}^{1} \lambda(x) \mathrm{d} x}=1-\frac{\sum_{j=2}^{d_{r}} \rho_{j} / j}{\sum_{j=2}^{d_{l}} \lambda_{j} / j}
$$

The degree distribution $(\lambda, \rho)$ from an edge perspective can be converted to a node perspective degree distribution $(L, R)$ defined by the following polynomials [13]

$$
\begin{aligned}
L(x) & =\sum_{i=2}^{d_{l}} L_{i} x^{i} \\
R(x) & =\sum_{j=2}^{d_{r}} R_{j} x^{j}
\end{aligned}
$$

with the following relations

$$
\begin{aligned}
& L_{j}=\frac{\lambda_{j}}{j \int_{0}^{1} \lambda(x)}=\frac{\lambda_{j} / j}{\sum_{i=2}^{d_{l}} \lambda_{i} / i} \\
& R_{j}=\frac{\rho_{j}}{j \int_{0}^{1} \rho(x)}=\frac{\rho_{j} / j}{\sum_{i=2}^{d_{r}} \rho_{i} / i}
\end{aligned}
$$

\section{WEIGHT DISTRIBUTION OF LDPC CODES}

In his doctoral dissertation [1], Gallager showed that, for a given regular LDPC code ensemble $\mathcal{C}\left(n, x^{j-1}, x^{k-1}\right)$, with block length $n$, degree $j \mathrm{v}$-nodes, and degree $k$ c-nodes, there exists a positive number $\delta$ such that at most a fraction $O\left(n^{-j+2}\right)$ of all codes have codewords of weight $\lfloor\delta n\rfloor$ or less, provided that $j$ is larger than 2 . If $j=2$, all codes in the ensemble have codewords of weight not exceeding $2+\frac{2 \ln (n / 2)}{\ln (k-1)}$.

It was also demonstrated in [13] that the fraction of degreetwo v-nodes plays a crucial role in the theory and practice of LDPC codes. In particular, the main result of [13] states that, for irregular LDPC codes ensembles, the growth rate of the minimum distance, i.e., whether this growth rate is linear or simply sublinear, depends only on $\lambda^{\prime}(0) \rho^{\prime}(1)$, a quantity which is related to the degree distributions (1) and (2). In particular, as shown in Section $\mathrm{V}, \lambda^{\prime}(0)$ is the fraction of edges connecting to degree-two v-nodes. As stated more precisely in 
Theorem 1 of [13], if $\lambda^{\prime}(0) \rho^{\prime}(1)>1$, the minimum distance grows sublinearly with the block length, otherwise, i.e., if $\lambda^{\prime}(0) \rho^{\prime}(1)<1$, it grows linearly with the block length, with probability at least $1-\ln \frac{1}{\sqrt{1-\lambda^{\prime}(0) \rho^{\prime}(1)}}$, sharpened to $\sqrt{1-\lambda^{\prime}(0) \rho^{\prime}(1)}$ in [14].

Moreover, the quantity $\lambda^{\prime}(0) \rho^{\prime}(1)$ also plays a fundamental role in the stability analysis of LDPC codes under iterative decoding, as well as in determining whether such codes are linear-time encodable. The stability condition of iterative decoders for LDPC codes, which was derived in [15], [16] through a density evolution analysis, states that the fundamental condition for a successful iterative decoding is given by the error probability, that needs to reach a sufficiently small value. For the binary erasure channel (BEC), it requires the condition $\lambda^{\prime}(0) \rho^{\prime}(1)<\frac{1}{\epsilon}$, being $\epsilon$ the channel parameter.

The "encoding" complexity of LDPC ensembles was investigated in [17] where it was shown that "optimized" LDPC ensembles admit linear time encoding and that $\lambda^{\prime}(0) \rho^{\prime}(1)>1$ is a sufficient condition for linear time encodable LDPC codes. Thus, it was pointed out in [13] that "the quantity $\lambda^{\prime}(0) \rho^{\prime}(1)$ connects three important code characteristics of LDPC codes:

1) performance,

2) encoding complexity,

3) and minimum distance."

The connections between all these features were well depicted in Figs. 1 and 2 of [13]. More precisely, Fig. 1 in [13] illustrates that capacity-achieving LDPC sequences over the BEC under certain technical conditions fulfill the inequality $\lambda^{\prime}(0) \rho^{\prime}(1)>1$, which is the case of interest of our paper. It is shown later in [13] that precisely this inequality also implies minimum distance sublinear growth. Moreover, as remarked earlier, it was shown in [17] that a standard LDPC ensemble needs to satisfy the condition $\lambda^{\prime}(0) \rho^{\prime}(1)>1$ to be linear time encodable.

A very interesting and general result connecting encoder complexity and minimum distance was given also in [19]. He proved that linear codes cannot have minimum distance growing linearly with the block length when the rate is nonvanishing if the encoder uses linear time and sublinear memory in the general binary branching program mode (parallel concatenated turbo codes [20]-[22] fall in this category). However, this result does not apply to standard LDPC codes since the memory of the encoder is not logarithmic.

Finally, in [13] it was shown that, for the case $\lambda^{\prime}(0) \rho^{\prime}(1)>$ 1 , which is the one examined in this paper (see the results reported in the next section), the expected number of codewords of weight $l$ "can be lower-bounded by the expected number of zigzag structures of size $2 l$ since every structure gives rise to a codeword of weight $l$ ". Thus, for any constant $l \leq \min \left\{L_{2} n,(1-r) n\right\}$, where $n$ is the codeword length, the probability of having no weight- $l$ codewords is given by

$$
\operatorname{Pr}\left(X_{l}=0\right)=\mathrm{e}^{-\frac{\left(\lambda^{\prime}(0) \rho^{\prime}(1)\right)^{l}}{2 l}}+O\left(n^{-1 / 3}\right)
$$

Thus, the probability that a randomly chosen code $\mathcal{G} \in$ $\mathcal{C}(n, \lambda, \rho)$ has at least one codeword of weight $l$ is given by
TABLE I

PARAMETERS OF GOOD RATE- $1 / 2$ CODES Listed IN TABle I OF [18] WITH MAXIMUM VARIABLE NODE DEGREES $d_{l}=4,5,6,7$, AND 8 .

\begin{tabular}{|c|c|c|c|c|c|}
\hline$d_{l}$ & 4 & 5 & 6 & 7 & 8 \\
\hline \hline$\lambda_{2}$ & 0.38354 & 0.32660 & 0.33241 & 0.31570 & 0.30013 \\
\hline$\lambda_{3}$ & 0.04237 & 0.11960 & 0.24632 & 0.41672 & 0.28395 \\
\hline$\lambda_{4}$ & 0.57409 & 0.18393 & 0.11014 & & \\
\hline$\lambda_{5}$ & & 0.36988 & & & \\
\hline$\lambda_{6}$ & & & 0.31112 & & \\
\hline$\lambda_{7}$ & & & & 0.43810 & \\
\hline$\lambda_{8}$ & & & & & 0.41592 \\
\hline \hline$\rho_{5}$ & 0.24123 & & & & \\
\hline$\rho_{6}$ & 0.75877 & 0.78555 & 0.76611 & 0.43810 & 0.22919 \\
\hline$\rho_{7}$ & & 0.21445 & 0.23389 & 0.56190 & 0.77081 \\
\hline \hline$\lambda^{\prime}(0)$ & 0.38354 & 0.32660 & 0.33241 & 0.31570 & 0.30013 \\
\hline$\rho^{\prime}(1)$ & 4.75877 & 5.21445 & 5.23389 & 5.56190 & 5.77081 \\
\hline \hline$\lambda^{\prime}(0) \rho^{\prime}(1)$ & 1.82518 & 1.70304 & 1.73980 & 1.75589 & 1.73199 \\
\hline \hline $\int_{0}^{1} \lambda(x)$ & 0.34942 & 0.32313 & 0.32770 & 0.35934 & 0.29671 \\
\hline$L_{2}$ & 0.54883 & 0.50538 & 0.50719 & 0.43927 & 0.50577 \\
\hline
\end{tabular}

$$
1-\operatorname{Pr}\left(X_{l}=0\right)=1-\mathrm{e}^{-\frac{\left(\lambda^{\prime}(0) \rho^{\prime}(1)\right)^{l}}{2 l}}+O\left(n^{-1 / 3}\right)
$$

In conclusion, both the parameter $L_{2}$, given by (6) with $j=2$, and the product $\lambda^{\prime}(0) \rho^{\prime}(1)$ are important in determining an LDPC code weight distribution and thus its performance.

\section{RESULTS ON WEIGHT DISTRIBUTIONS}

Being

$$
\lambda^{\prime}(0)=\left.\sum_{i=2}^{d_{l}}(i-1) \lambda_{i} x^{i-2}\right|_{x=0}=\lambda_{2}
$$

and

$$
\rho^{\prime}(1)=\left.\sum_{j=2}^{d_{r}}(j-1) \rho_{j} x^{j-2}\right|_{x=1}=\sum_{j=2}^{d_{r}}(j-1) \rho_{j}
$$

we obtain

$$
\lambda^{\prime}(0) \rho^{\prime}(1)=\lambda_{2} \sum_{j=2}^{d_{r}}(j-1) \rho_{j}
$$

The products $\lambda^{\prime}(0) \rho^{\prime}(1)$ have been computed for the rate$1 / 2$ irregular LDPC codes found in [18] (see Tables I and II of [18]) together with the parameters $L_{2}$. Both are reported in Tables I and II. As may be seen from these tables, the values of the products $\lambda^{\prime}(0) \rho^{\prime}(1)$ are all greater than 1 and there is a general decreasing of these values while increasing $d_{l}$, even if this decreasing presents some irregularities. On the other hand, there is a general decreasing of the $L_{2}$ values while increasing $d_{l}$, even if also in this case this decreasing presents some irregularities. 
TABLE II

PARAMETERS OF GOOD RATE- $1 / 2$ CODES LISTED IN TABLE I OF [18] WITH MAXIMUM VARIABLE NODE DEGREES $d_{l}=9,10,11$, AND 12 .

\begin{tabular}{|c|c|c|c|c|}
\hline$d_{l}$ & 9 & 10 & 11 & 12 \\
\hline \hline$\lambda_{2}$ & 0.27684 & 0.25105 & 0.23882 & 0.24426 \\
\hline$\lambda_{3}$ & 0.28342 & 0.30938 & 0.29515 & 0.25907 \\
\hline$\lambda_{4}$ & & 0.00104 & 0.03261 & 0.01054 \\
\hline$\lambda_{5}$ & & & & 0.05510 \\
\hline$\lambda_{6}$ & & & & \\
\hline$\lambda_{7}$ & & & & \\
\hline$\lambda_{8}$ & & & & 0.01455 \\
\hline$\lambda_{9}$ & 0.43974 & & & \\
\hline$\lambda_{10}$ & & 0.43853 & & 0.01275 \\
\hline$\lambda_{11}$ & & & 0.43342 & \\
\hline$\lambda_{12}$ & & & & 0.40373 \\
\hline \hline$\rho_{6}$ & 0.01568 & & & \\
\hline$\rho_{7}$ & 0.85244 & 0.63676 & 0.43011 & 0.25475 \\
\hline$\rho_{8}$ & 0.13188 & 0.36324 & 0.56989 & 0.73438 \\
\hline$\rho_{9}$ & & & & 0.01087 \\
\hline \hline$\lambda^{\prime}(0)$ & 0.27684 & 0.25105 & 0.23882 & 0.24426 \\
\hline$\rho^{\prime}(1)$ & 6.11620 & 6.36324 & 6.56989 & 6.75612 \\
\hline \hline$\lambda^{\prime}(0) \rho^{\prime}(1)$ & 1.69321 & 1.59749 & 1.56902 & 1.65025 \\
\hline \hline $\int_{0}^{1} \lambda(x)$ & 0.28175 & 0.27276 & 0.26535 & 0.25888 \\
\hline$L_{2}$ & 0.49128 & 0.46020 & 0.45001 & 0.47176 \\
\hline
\end{tabular}

\section{Simulation RESUlts}

Consider an ensemble of random codes with edgeperspective degree distributions $\lambda(x)$ and $\rho(x)$ given in Tables I and II. A custom software based on [23] (also used in [24] and [25] to design well performing rate compatible puncturing patterns for LDPC $\operatorname{codes}^{1}$ on the basis of the results of [28] and [29]) was employed to simulate their performance over an AWGN channel, assuming a BPSK (Binary Phase Shift Keying) modulator. The belief propagation algorithm, also called message passing or sum-product algorithm, commonly employed for LDPC decoding, has been adopted, employing soft decision.

From (9), giving the probability that a randomly chosen code $\mathcal{G} \in \mathcal{C}(n, \lambda, \rho)$ has at least one codeword of weight $l$, and being $l \leq \min \left\{L_{2} n,(1-r) n\right\}$, two cases should be distinguished:

1) $L_{2}>(1-r)$ implying $l \leq(1-r) n$

2) $L_{2}<(1-r)$ implying $l \leq L_{2} n$

\section{A. Case 1: $L_{2}>(1-r)$}

Being, for the codes shown in Tables I and II, the rate $r=1 / 2$, and thus $1-r=1 / 2$, the first case, i.e., $L_{2}>0.5$, is encountered, e.g., by the codes with $d_{l}=4,6$, and 8 of Table I. This implies that $l \leq(1-r) n=0.5 n$, i.e., that $l$ is upper bounded by the constant $0.5 \mathrm{n}$. Being this upper bound on $l$ the same for all the 3 above mentioned codes (i.e., those with

${ }^{1}$ Useful in Automatic Repeat-reQuest / Forward Error Correction (ARQ / FEC) schemes [26] and for Unequal Error Protection (UEP) applications [27].

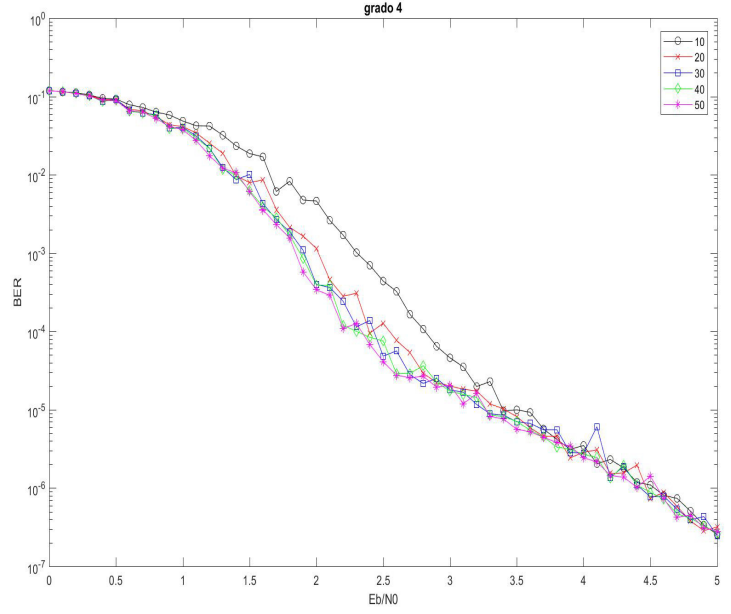

Fig. 1. BER vs. $E_{b} / N_{0}$ in $\mathrm{dB}$ of rate- $1 / 2$ randomly chosen codes with distribution pairs $(\lambda, \rho)$ with $d_{l}=4$ (given in the 2-nd column of Table I) and $n=1000$. The performance curves have been obtained with an iteration number $I=10,20,30,40$, and 50 of the decoding algorithm.

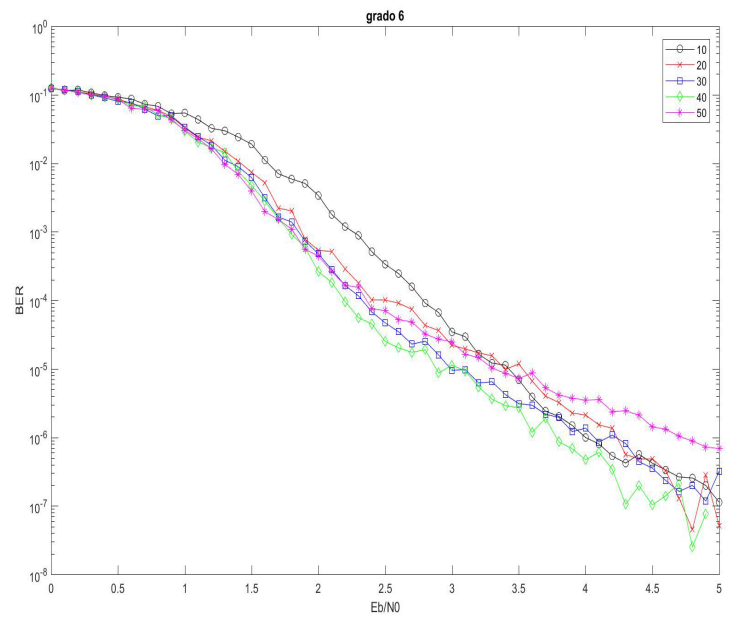

Fig. 2. BER vs. $E_{b} / N_{0}$ in $\mathrm{dB}$ of rate- $1 / 2$ randomly chosen codes with distribution pairs $(\lambda, \rho)$ with $d_{l}=6$ (given in the 4-th column of Table I) and $n=1000$. The performance curves have been obtained with an iteration number $I=10,20,30,40$, and 50 of the decoding algorithm.

$d_{l}=4,6$, and 8 of Table I), the value of the product $\lambda^{\prime}(0) \rho^{\prime}(1)$ alone makes the difference in this case. In particular, looking at (9), the lower is the value of the product $\lambda^{\prime}(0) \rho^{\prime}(1)$, the higher the value assumed by the probability (8), and thus the lower the value assumed by the probability (9), i.e., the lower is the probability that a randomly chosen code $\mathcal{G} \in \mathcal{C}(n, \lambda, \rho)$ has at least one codeword of weight $l \leq 0.5 n$. Thus, a randomly chosen code with distribution pairs $(\lambda, \rho)$ given in the 6-th column of Table I (with $d_{l}=8$ ), since it presents a value of the product $\lambda^{\prime}(0) \rho^{\prime}(1)=1.73199$, will have a lower probability of presenting at least one codeword of weight $l \leq 0.5 n$ than a randomly chosen code with distribution pairs $(\lambda, \rho)$ 


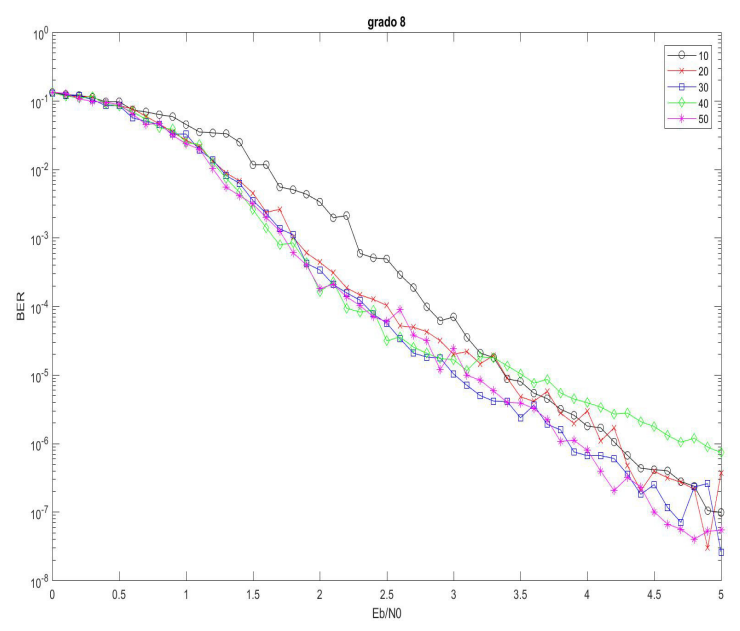

Fig. 3. BER vs. $E_{b} / N_{0}$ in $\mathrm{dB}$ of rate-1/2 randomly chosen codes with distribution pairs $(\lambda, \rho)$ with $d_{l}=8$ (given in the 6-th column of Table I) and $n=1000$. The performance curves have been obtained with an iteration number $I=10,20,30,40$, and 50 of the decoding algorithm.

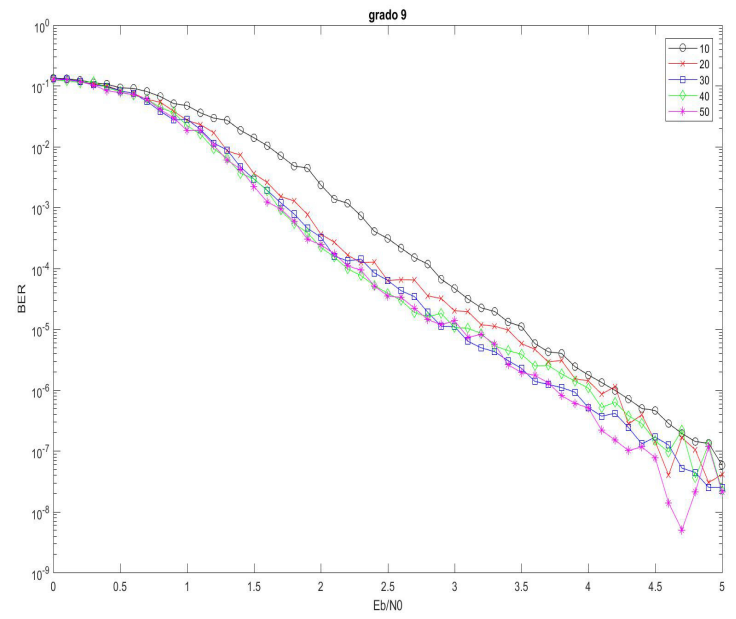

Fig. 4. BER vs. $E_{b} / N_{0}$ in $\mathrm{dB}$ of rate-1/2 randomly chosen codes with distribution pairs $(\lambda, \rho)$ with $d_{l}=9$ (given in the 1 -st column of Table II) and $n=1000$. The performance curves have been obtained with an iteration number $I=10,20,30,40$, and 50 of the decoding algorithm.

given in the 4-th column of Table I (with $d_{l}=6$ ), since the latter presents a higher value of the product $\lambda^{\prime}(0) \rho^{\prime}(1)$ (i.e., $\left.\lambda^{\prime}(0) \rho^{\prime}(1)=1.73980\right)$. The same may be said when comparing, e.g., a randomly chosen code with distribution pairs $(\lambda, \rho)$ with $d_{l}=6$ (given in the 4-th column of Table I), and a randomly chosen code with distribution pairs $(\lambda, \rho)$ with $d_{l}=4$ (given in the 2-nd column of Table I).

In Figs. 1, 2, and 3 are shown the BER performance curves of some randomly chosen codes with distribution pairs $(\lambda, \rho)$ shown in the 2-nd, 4-th and 6-th column of Table I, i.e., with $d_{l}=4,6$, and 8 , respectively.

As expected from the above described analysis, the BER

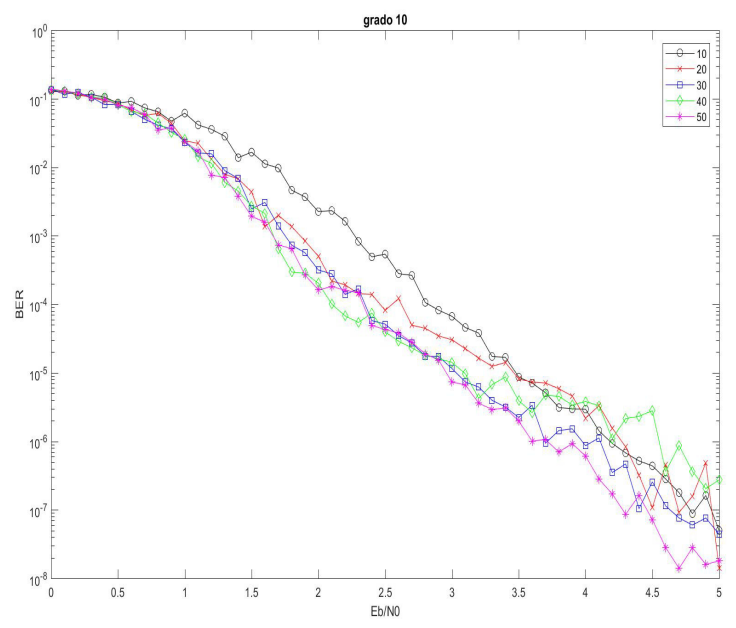

Fig. 5. BER vs. $E_{b} / N_{0}$ in $\mathrm{dB}$ of rate- $1 / 2$ randomly chosen codes with distribution pairs $(\lambda, \rho)$ with $d_{l}=10$ (given in the 2-nd column of Table II) and $n=1000$. The performance curves have been obtained with an iteration number $I=10,20,30,40$, and 50 of the decoding algorithm.

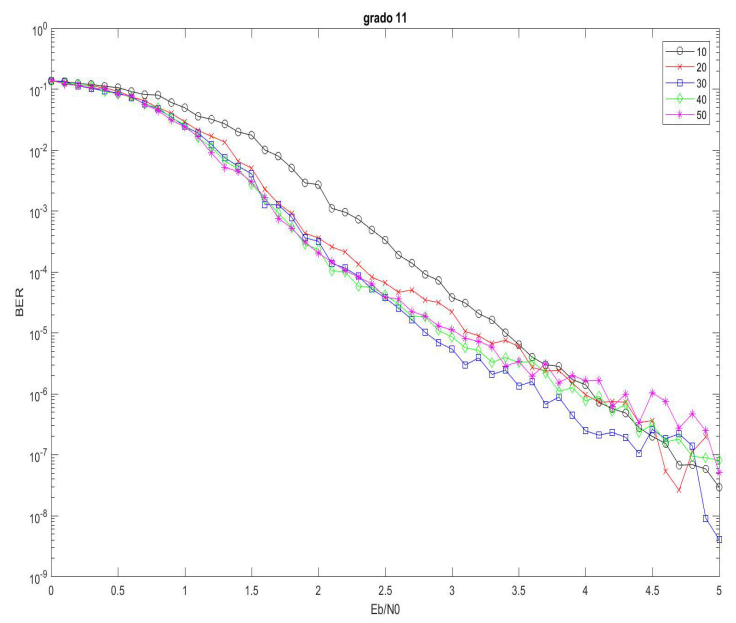

Fig. 6. BER vs. $E_{b} / N_{0}$ in $\mathrm{dB}$ of rate-1/2 randomly chosen codes with distribution pairs $(\lambda, \rho)$ with $d_{l}=11$ (given in the 3 -rd column of Table II) and $n=1000$. The performance curves have been obtained with an iteration number $I=10,20,30,40$, and 50 of the decoding algorithm.

performance obtained with $d_{l}=6$ (Fig. 2) is better than that obtained with $d_{l}=4$ (Fig. 1), and the BER performance obtained with $d_{l}=8$ (Fig. 3 ) is the best of the three.

\section{B. Case 2: $L_{2}<(1-r)$}

Being, for the codes shown in Tables I and II, the rate $r=$ $1 / 2$, and thus $1-r=1 / 2$, the second case, i.e., $L_{2}<0.5$, is encountered, e.g., by the codes with $d_{l}=9,10$, and 11 of Table II. This implies that $l \leq L_{2} n$, i.e., that $l$ is upper bounded by a constant depending on the $L_{2}$ value. Being this upper bound on $l$ different for the 3 above mentioned codes (i.e., those with $d_{l}=9,10$, and 11 of Table II), the value of the 
product $\lambda^{\prime}(0) \rho^{\prime}(1)$ alone should not make the difference, in this case, since a lower upper bound on the codeword weight $l$ could lead to a worse BER performance. But, looking at Figs. 4, 5, and 6 where are presented the BER performance curves of some randomly chosen codes with distribution pairs $(\lambda, \rho)$ shown in the 1-st, 2-nd and 3-rd column of Table II, i.e., with $d_{l}=9,10$, and 11 , respectively, the BER performance obtained with $d_{l}=10$ (Fig. 5) is better than that obtained with $d_{l}=9$ (Fig. 4), since the codes with $d_{l}=10$ present a lower $L_{2}$ value $\left(L_{2}=0.46020\right)$ than those with $d_{l}=9\left(L_{2}=\right.$ 0.49128 ), thus a lower upper bound on $l$, but also present a lower value of the product $\lambda^{\prime}(0) \rho^{\prime}(1)(1.59749$ vs. 1.69321$)$ which, however, leads to a better performance, as already said in the previous subsection, even if the upper bound on the codeword weight $l$ is worse. The BER performance obtained with $d_{l}=11$ (Fig. 6) is the best of the three since it presents the lowest value of $L_{2}(0.45001)$ but also the lowest value of the product $\lambda^{\prime}(0) \rho^{\prime}(1)(1.56902)$.

\section{CONCLUSIONS}

This paper was focused on the role played by the product $\lambda^{\prime}(0) \rho^{\prime}(1)$, quantity related to the degree distributions (1) and (2), in determining the performance of an LDPC code. As stated in Theorem 1 of [13], if $\lambda^{\prime}(0) \rho^{\prime}(1)>1$, the minimum distance grows sublinearly with the block length, otherwise, i.e., if $\lambda^{\prime}(0) \rho^{\prime}(1)<1$, it grows linearly with the block length, with probability at least $1-\ln \frac{1}{\sqrt{1-\lambda^{\prime}(0) \rho^{\prime}(1)}}$, sharpened to $\sqrt{1-\lambda^{\prime}(0) \rho^{\prime}(1)}$ in [14]. This paper was focused on the codes for which $\lambda^{\prime}(0) \rho^{\prime}(1)>1$, showing that the lower is the value of this product, the better is the BER code performance. In particular, the analysis was performed for two principal cases, i.e., the one for which the $L_{2}$ value (given by (6) with $j=2$ ) is higher than $(1-r)$ (being $r$ the code rate) and the one for which $L_{2}$ is lower than $(1-r)$.

\section{REFERENCES}

[1] Robert G. Gallager, "Low-density parity-check codes", MIT press Cambridge, 1963

[2] R. M. Tanner, "A recursive approach to low complexity codes", IEEE Transactions on Information Theory, vol. 27, no. 5, September 1981, pp. 533-547.

[3] David J. C. Mackay, "Good error correcting codes based on very sparse matrices", IEEE Transactions on Information Theory, vol. 45, no. 2, March 1999, pp. 399-431.

[4] M. G. Luby, M. Mitzenmacher, M. A. Shokrollahi, and D. A. Spielman, "Analysis of Low Density Codes and improved design using Irregular Graphs", IEEE Transactions on Information Theory, vol. 47, no. 2, February 2001, pp. 585-598.

[5] F. Babich, A. Crismani, M. Driusso, and L. Hanzo, "Design criteria and genetic algorithm aided optimization of three-stage-concatenated spacetime shift keying systems", IEEE Signal Processing Letters, vol. 19, no. 8, August 2012, pp. 543-546.

[6] F. Babich, M. Comisso, and R. Corrado, "On the impact of the video quality assessment in $802.11 \mathrm{e}$ ad-hoc networks using adaptive retransmissions", Proc. of the IEEE IFIP Annual Mediterranean Ad Hoc Networking Workshop, Piran, Slovenia, June 2-4, 2014, pp. 1-8.

[7] F. Babich, M. Comisso, A. Crismani, and A. Dorni, "On the design of MAC protocols for multi-packet communication in IEEE 802.11 heterogeneous networks using adaptive antenna arrays", IEEE Transactions on Mobile Computing, vol. 14, no. 11, November 2015, pp. 2332-2348.
[8] V. Sidorenko, M. Bossert, and F. Vatta, "Properties and encoding aspects of direct product convolutional codes", Proc. of the 2012 IEEE International Symposium on Information Theory, ISIT'12, Boston, U.S.A., July 1-6, 2012, pp. 2351-2355.

[9] B. Liu, Y. Li, B. Rong, L. Gui, and Y. Wu, "LDPC-RS Product Codes for Digital Terrestrial Broadcasting Transmission System", IEEE Trans. on Broadcasting, vol. 60, no. 1, March 2014, pp. 38-49.

[10] M. Comisso and F. Babich, "Coverage analysis for 2D/3D millimeter wave peer-to-peer networks", IEEE Transactions on Wireless Communications, vol. 18, no. 7, July 2019, pp. 3613-3627.

[11] F. Babich and M. Comisso, "Impact of segmentation and capture on slotted Aloha systems exploiting interference cancellation", IEEE Trans. on Vehicular Technology, vol. 68, no. 3, March 2019, pp. 2878-2892.

[12] F. Babich and M. Comisso, "Including the angular domain in the analysis of finite multi-packet peer-to-peer networks with uniformly distributed sources", IEEE Transactions on Communications, vol. 64, no. 6, June 2016, pp. 2494-2510.

[13] C. Di, T. J. Richardson, and R. Urbanke, "Weight Distribution of Low-Density Parity-Check Codes", IEEE Transactions on Information Theory, vol. 52, no. 11, Nov. 2006, pp. 4839-4855.

[14] A. Orlitsky, K. Viswanathan, and J. Zhang, "Stopping set distribution of LDPC code ensembles", IEEE Transactions on Information Theory, vol. 51, no. 3, Mar. 2005, pp. 929-953.

[15] T. J. Richardson, M. A. Shokrollahi, and R. L. Urbanke, "Design of provably good low-density parity check codes", IEEE Transactions on Information Theory, vol. 47, no. 2, February 2001, pp. 599-618.

[16] A. Shokrollahi, "New sequences of linear time erasure codes approaching the channel capacity", Proc. of AAECC-13 (Lecture Notes in Computer Science), Berlin, Germany, vol. 1719, 1999, pp. 65-76.

[17] T. Richardson and R. Urbanke, "Efficient encoding of low-density paritycheck codes", IEEE Transactions on Information Theory, vol. 47, no. 2, February 2001, pp. 638-656.

[18] T. J. Richardson, M. A. Shokrollahi, and R. L. Urbanke, "Design of Capacity-Approaching Irregular Low-Density Parity-Check Codes", IEEE Transactions on Information Theory, vol. 47, no. 2, February 2001, pp. 619-637.

[19] L. M. Bazzi, "Minimum Distance of Error Correcting Codes Versus Encoding Complexity, Symmetry, and Pseudorandomness", Ph.D. dissertation, MIT, Cambridge, MA, 2003.

[20] C. Berrou, A. Glavieux, and P. Thitimajshima, "Near Shannon limit error-correcting coding and decoding", Proc. of the IEEE Int. Conf. on Communications, Geneva, Switzerland, May 1993, pp. 1064-1070.

[21] F. Babich, G. Montorsi and F. Vatta, "On rate-compatible punctured turbo codes (RCPTC) design", EURASIP Journal on Applied Signal Processing, no. 6, May 2005, pp. 784-794.

[22] C. Koller, A. Graell i Amat, J. Kliewer, F. Vatta, K. Sh. Zigangirov, and D. J. Costello, Jr., "Analysis and design of tuned turbo codes", IEEE Transactions on Information Theory, vol. 58, no. 7, July 2012, pp. 4796-4813.

[23] A. Boscolo, F. Vatta, F. Armani, E. Viviani, and D. Salvalaggio,"Physical AWGN channel emulator for Bit Error Rate test of digital baseband communication", Applied Mechanics and Materials, Vols. 241-244, 2013, pp. 2491-2495.

[24] F. Babich, M. Noschese, and F. Vatta, "Analysis and design of rate compatible LDPC codes", Proc. of the 27th IEEE International Symposium on Personal, Indoor and Mobile Radio Communications, PIMRC'16, Valencia, Spain, September 4-8, 2016, pp. 1-6.

[25] F. Babich, M. Noschese, A. Soranzo, and F. Vatta, "Low complexity rate compatible puncturing patterns design for LDPC codes", Proc. of the 2017 Int. Conf. SoftCOM, Split, Croatia, Sept. 21-23, 2017.

[26] F. Babich and F. Vatta, "Turbo codes construction for robust hybrid multitransmission schemes", Journal of Communication Software and Systems (JCOMSS), vol. 7, no. 4, December 2011, pp. 128-135.

[27] F. Babich, M. D'Orlando, and F. Vatta, "Distortion estimation algorithms for real-time video streaming: an application scenario", Proc. of the 2011 Int. Conf. on Software, Telecommunications and Computer Networks, SoftCOM'11, Split, Croatia, September 15-17, 2011, pp. 291-295.

[28] F. Babich, A. Soranzo, and F. Vatta, "Useful mathematical tools for capacity approaching codes design", IEEE Communications Letters, vol. 21, no. 9, Sept. 2017, pp. 1949-1952.

[29] F. Vatta, A. Soranzo, and F. Babich, "Low-Complexity bound on irregular LDPC belief-propagation decoding thresholds using a Gaussian approximation", Electronics Letters, vol. 54, no. 17, Aug. 2018, pp. 1038-1040. 